\title{
The Relationship between Conidial Germination and Esterase Activities in Neurospora crassa
}

\author{
By TAKESHI EGASHIRA \\ Department of Biology, Kyushu Dental College, Kitakyushu 803, Japan
}

(Received 20 July 1982; revised 18 December 1982)

\begin{abstract}
Esterase activity in rapidly germinating Neurospora conidia was several times higher than the esterase activity in conidia which germinate slowly. Starch gel electrophoresis experiments demonstrated the existence of esterase isoenzymes which are specific to the conidia. These isoenzymes completely disappeared during $20 \mathrm{~h}$ of conidial germination at $30^{\circ} \mathrm{C}$. Electron microscopy showed the successive breakdown of electron-dense compounds in storage bodies during conidial germination. These observations, taken together, indicate that the electrondense compounds may be hydrolysed by specific esterases to serve as an endogenous energy and material source for germ tube formation. The levels of esterase activity, however, were not always proportional to the time required for conidial germination, indicating the possibility that additional enzyme systems might also be involved in the initial stages of germination.
\end{abstract}

\section{INTRODUCTION}

Neurospora conidia begin to germinate after about $90 \mathrm{~min}$ when incubated in minimal medium at $30^{\circ} \mathrm{C}$. Many biochemical events have been demonstrated to occur prior to germ tube formation (Schmit \& Brody, 1976). According to the reports of Mirkes and his co-workers, an exogenous carbon source as well as some inorganic salts (magnesium and zinc) are essential for the emergence of conidial germ tubes (Mirkes, 1974, 1977; Farach et al., 1979).

Weiss (1963) reported the presence in Neurospora conidia of vesicles containing electrondense compounds, which might be lipid, and Bianchi \& Turian (1967) observed a rapid decrease in the lipid content during the germ tube elongation. The rapid decrease may be due to the degradation of the putative lipid compounds present in the storage vesicles.

If these endogenous compounds are utilized for germ tube formation before the onset of nutrient uptake from the growth medium, high levels of some hydrolytic enzymes may be available in conidia for rapid formation of germ tubes, especially at the early stages of germination. Preliminary observations showed that conidia of strain 4A could germinate earlier than those of strain $38 \mathrm{a}$. The present study was designed to compare the relationship between esterase activity and time of conidial germination in these two strains. Changes in subcellular structures were also followed during the course of conidial germination.

\section{METHODS}

Strains and conidial preparation. The strains used in this study were $4 \mathrm{~A}, 38 \mathrm{a}$, and the progeny from matings between them: cultures 22, 32, 62, 47,64 and 80. Strain 4A (FGSC 821) is wild type with respect to conidial pigmentation, hyphal morphogenesis, and nutrient requirement, but has the mutant gene tan which yields yellowish-brown ascospores in matings. Strain 38a (FGSC 38) is also wild-type in all respects, except for carrying the pe gene which is responsible for the formation of 'peach' conidia.

Conidia were harvested from cultures grown for 7 to $10 \mathrm{~d}$ at $27^{\circ} \mathrm{C}$ on minimal media (Beadle \& Tatum, 1945) by filtering through glass-wool and washing four times in distilled water. These conidia were used for the experiments after 1 to $3 \mathrm{~d}$ storage at $-17^{\circ} \mathrm{C}$. 
Germination test. Conidia were resuspended in minimal medium $(1 \mathrm{ml})$ at a concentration of $10^{6}$ to $10^{7}$ conidia $\mathrm{ml}^{-1}$ and shaken vigorously at $30^{\circ} \mathrm{C}$. Samples were withdrawn from the conidial suspensions at definite intervals during the first $6 \mathrm{~h}$ of incubation to investigate the frequency of germination.

Preparation of cell-free extracts. Cell-free extracts were prepared by conidial disruption with an Edmund Bühler Vibriogen cell mill, followed by centrifugation at $10000 \mathrm{~g}$ for $30 \mathrm{~min}$.

Assays of esterase activity. Esterase activity was measured using $0.9 \mathrm{M}$-borate buffer (pH 8.0) containing 19 mMn-butyric acid ethyl ester and $13 \mu \mathrm{M}$-cresol red according to the procedure described in the Biochemicals Catalogue of Boehringer. The reaction mixture contained $3 \mathrm{ml}$ of the above mixture and $0 \cdot 1 \mathrm{ml}$ cell-free extract. Since the acid formed during the reaction discolours cresol red, the rate of decrease in absorbance at $578 \mathrm{~nm}$ was measured for an initial $5 \mathrm{~min}$ at $27^{\circ} \mathrm{C}$. Relative activity of esterase was given by the rate of decrease in absorbance in $1 \mathrm{~min}$ (mg protein $)^{-1}$. Protein was measured by the Lowry method using BSA as the standard.

Electrophoresis. Starch gel electrophoresis was carried out as described by Markert \& Faulhaber (1965) using Tris/EDTA/borate buffer ( $\mathrm{pH} 8.7$ ). The reaction mixture for visualization of enzyme activity contained $4 \mathrm{ml} 2 \%$ (w/v) $\alpha$-naphthyl acetate in acetone, $300 \mathrm{ml} \mathrm{0.05} \mathrm{M-phosphate} \mathrm{buffer} \mathrm{(pH} \mathrm{7.0)} \mathrm{and} 100 \mathrm{mg}$ Fast Blue RR. Hyphae, for the comparative studies of esterases in conidia and hyphae by zymograms, were produced by germinating conidia in minimal liquid medium for $20 \mathrm{~h}$ at $30^{\circ} \mathrm{C}$. A cell-free extract of the resultant mycelial mat was used for the electrophoresis.

The esterase inhibitor diisopropyl fluorophosphate was used by pre-incubating the starch gel in a solution of the inhibitor in $0.05 \mathrm{M}$-phosphate buffer ( $\mathrm{pH} 7 \cdot 0$ ) for $30 \mathrm{~min}$. The starch gel was then placed in the esterase reaction mixture to which the inhibitor also had been added. For examining the effect of heat on esterase activity, the extracts were subjected to the various heat treatments before electrophoresis.

Electron microscopy. For electron microscopy, germinating conidia were fixed in $6 \%(\mathrm{v} / \mathrm{v})$ aqueous glutaraldehyde followed by post-fixation in $2 \%(\mathrm{w} / \mathrm{v})$ aqueous $\mathrm{KMnO}_{4}$. To demonstrate acid phosphatase activity, cells were pre-fixed for $1 \mathrm{~h}$ in $0.2 \mathrm{M}$-cacodylate buffer $(\mathrm{pH} 7 \cdot 2)$ containing $2 \%(\mathrm{w} / \mathrm{v})$ paraformaldehyde and $2.5 \%$ glutaraldehyde. The samples were then rinsed in the medium of Barka \& Anderson (1962) with sodium $\beta$ glycerophosphate as a substrate and incubated for $2 \mathrm{~h}$ at $32{ }^{\circ} \mathrm{C}$. After incubation, the samples were post-fixed in $2 \%$ paraformaldehyde $/ 2.5 \%$ glutaraldehyde for $2.5 \mathrm{~h}$ and then in $2 \% \mathrm{OsO}_{4}$ for $14 \mathrm{~h}$.

\section{RESULTS \\ Conidial germination}

The results in Table 1 show changes of germination rate of conidia as a function of incubation period, along with data concerning volume of conidia and their esterase activities. Cultures 22 , $32,62,47,64$ and 80 are progeny from crosses between $4 \mathrm{~A}$ (early germination) and 38a (late germination). Conidia from $4 A$ and cultures 47,64 and 80 began to germinate about $60 \mathrm{~min}$ after the inoculation, and incubation for $4 \mathrm{~h}$ was enough to obtain more than $90 \%$ germination, whereas conidia from 38a and cultures 22,32 and 62 began to germinate 90 min after inoculation, and more than $4 \mathrm{~h}$ was required to obtain $90 \%$ germination. The conidia which showed early germination, however, did not always extend their germ tubes rapidly. For example, germ tube lengths of conidia from cultures 62,64 and 80 were all more than 10 times their respective conidial diameter after $5 \mathrm{~h}$ incubation even though germ tube initiation by conidia from culture 62 was slower than that of conidia from cultures 64 and 80 . The results suggest that germ tube emergence and germ tube elongation are controlled by different mechanisms.

The data also indicated that conidial germination percentages were not always proportional to the conidial volumes. Among the cultures examined, conidia from cultures 47 and 80 were largest, particularly those of culture 47 which were two to three times larger than those of other cultures. Despite the larger volume, conidia of culture 47 showed little difference in germination percentage from those of cultures $4 \mathrm{~A}$ and 64 whose conidia were much smaller. Within a single strain, however, larger conidia were more likely to germinate rapidly than smaller conidia (data not shown).

\section{Esterase activity in conidia}

If lipids are used as an endogenous source of energy and materials to produce germ tubes, one might expect conidia which germinate early to have high levels of esterase activity. Accordingly, the enzyme activities were measured using n-butyric acid ethyl ester as a substrate. The relative 
Table 1. Conidial volume, esterase activity, and percentage germination during the first $6 \mathrm{~h}$ of incubation at $30^{\circ} \mathrm{C}$

$\begin{array}{cc}\text { Culture* } & \begin{array}{c}\text { Volume of } \\ \text { conidia }\left(\mu \mathrm{m}^{3}\right) \dagger\end{array} \\ 4 \mathrm{~A} & 75 \pm 30 \\ 47 & 201 \pm 133 \\ 64 & 84 \pm 51 \\ 80 & 120 \pm 76 \\ 38 \mathrm{a} & 72 \pm 27 \\ 22 & 69 \pm 29 \\ 32 & 82 \pm 30 \\ 62 & 79 \pm 32\end{array}$

\author{
Relative activity \\ of esterase:
}

1
$2 \cdot 2$
$2 \cdot 4$
$0 \cdot 9$
$0 \cdot 4$
$0 \cdot 3$
$0 \cdot 5$
$0 \cdot 5$

Percentages of conidia with germ tubes after:

$\begin{array}{cccc}\overbrace{1.5 \mathrm{~h}} & 2.5 \mathrm{~h} & 4 \mathrm{~h} & 6 \mathrm{~h} \\ 5.5 & 13 \cdot 0 & >90 & >90 \\ 5 \cdot 5 & 18 \cdot 0 & >90 & >90 \\ 5 \cdot 0 & 32.5 & >90 & >90 \\ 6 \cdot 0 & 37.5 & >90 & >90 \\ 2 \cdot 5 & 5 \cdot 5 & 65 & >90 \\ 2 \cdot 5 & 5 \cdot 0 & 65 & >90 \\ 3.0 & 6.5 & 60 & >90 \\ 4.5 & 8.5 & 70 & >90\end{array}$

* Strain $4 \mathrm{~A}$ is an early-germinating strain and $38 \mathrm{a}$ is late-germinating; cultures $47,64,80,22,32$ and 62 are progeny derived from matings between them.

$\dagger$ Volume was calculated by estimating the length and width of conidia and by assuming their shape to be ellipsoidal; 150 conidia were measured from each culture. The values given are the mean \pm S.D.

$\ddagger$ Activity in strain 4A was taken as 1 . See Methods for details of the enzyme assay.

(a)

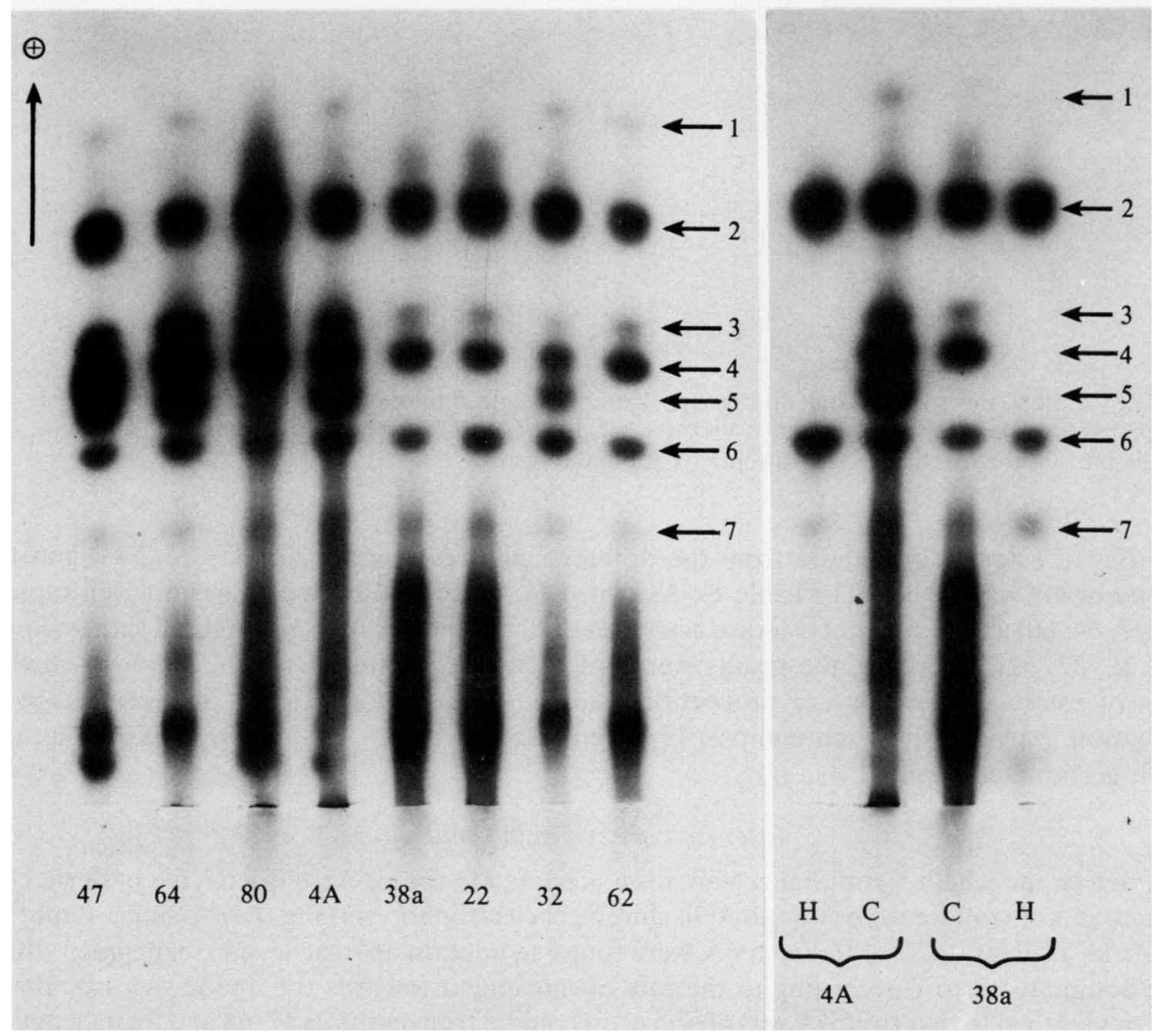

Fig. 1. Esterase zymograms in Neurospora. ( $a$ ) Isoenzyme patterns in conidia of strains $4 \mathrm{~A}, 38 \mathrm{a}$ and their progeny: cultures $47,64,80,22,32$ and 62 . Culture 62 is a recombinant type with regard to bands 1 and 5. Conidia of cultures $47,64,80$ and $4 \mathrm{~A}$ form germ tubes earlier than those of other cultures. $(b)$ Comparison of isoenzyme patterns in conidia (C) and $20 \mathrm{~h}$ germinants $(\mathrm{H})$. Isoenzymes corresponding to bands 1, 3, 4 and 5 completely disappeared in germinants of both cultures $4 \mathrm{~A}$ and $38 \mathrm{a}$. 


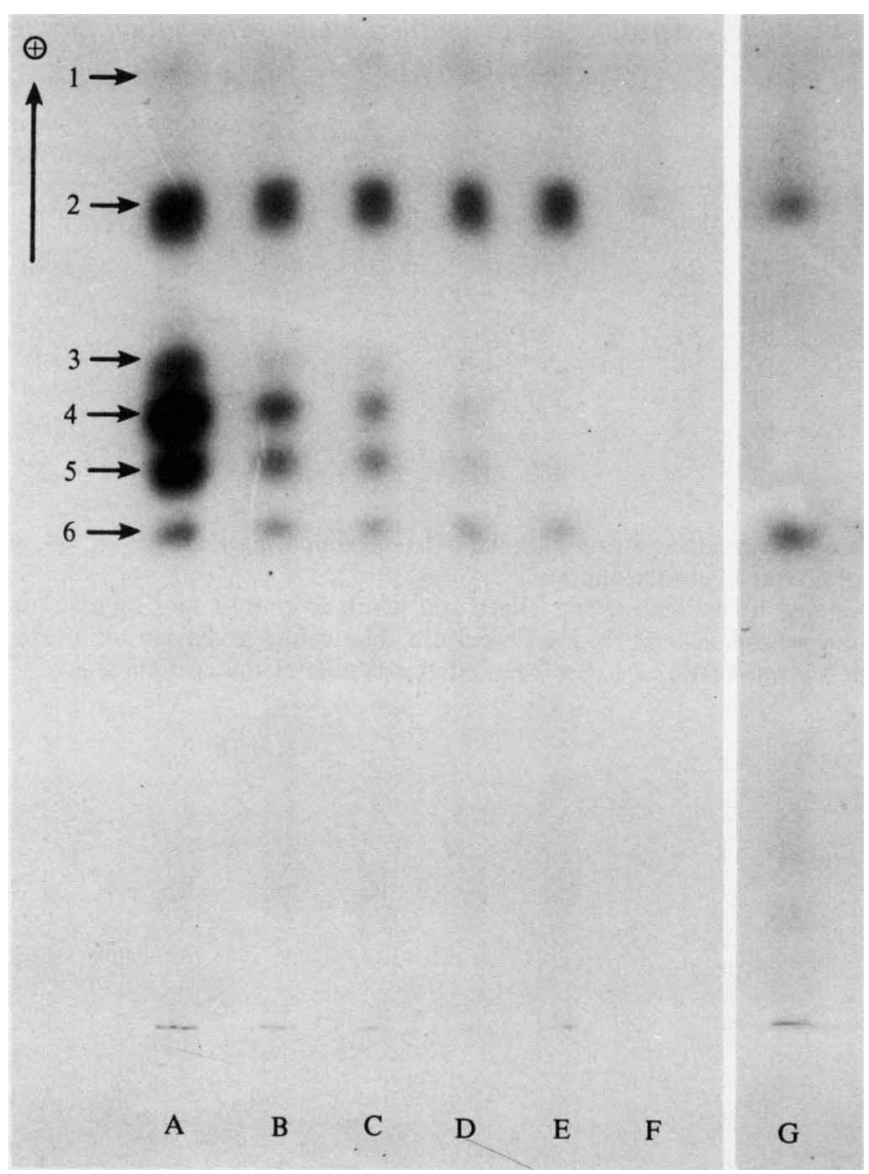

Fig. 2. Heat inactivation and diisopropyl fluorophosphate inhibition (strain 4A). A, Untreated preparation; B, C, D and $\mathrm{E}$, heated at $40^{\circ} \mathrm{C}$ for $5,7,10$ and $20 \mathrm{~min}$, respectively; $\mathrm{F}$, heated at $60^{\circ} \mathrm{C}$ for $20 \mathrm{~min} ; \mathrm{G}$, treated with $10^{-4} \mathrm{M}$-diisopropyl fluorophosphate.

activities of esterase in conidia from the different cultures ranged from 0.3 to 2.4 when the activity of $4 \mathrm{~A}$ was taken as 1 (Table 1). As expected, those conidia which germinated rapidly $(4 \mathrm{~A}, 47,64,80)$ showed higher esterase activities than did those which germinated rather slowly $(38 \mathrm{a}, 22,32,62)$. However, the results were not entirely consistent with the supposition that levels of esterase activities are proportional to the germination rate at the early stage of incubation, particularly when comparisons were made between conidia from those cultures which germinated rapidly.

\section{Esterase isoenzymes in conidia}

To determine whether conidial germination is related to the esterase isoenzyme pattern, cellfree extracts of conidia were submitted to starch gel electrophoresis (Fig. 1a). Using $\alpha$-naphthyl acetate as a substrate, conidia from $4 \mathrm{~A}$ were found to contain at least seven isoenzymes which were designated 1 to 7 according to the rate of movement towards the anode. An isoenzyme pattern identical to that from $4 \mathrm{~A}$ was observed in conidia from cultures 47,64 and 32 . Isoenzyme bands 1 and 5 were not detected in conidia from 38a, 22 and 80 , and conidia from 62 did not give band 5 . The results failed to show any correlation between a specific isoenzyme and early germination.

Esterase zymograms of cell-free extracts of mycelia $(20 \mathrm{~h}$ conidial germinants) were characterized by the disappearance of isoenzymes 1, 3, 4 and 5 in 4A, and isoenzymes 3 and 4 in 

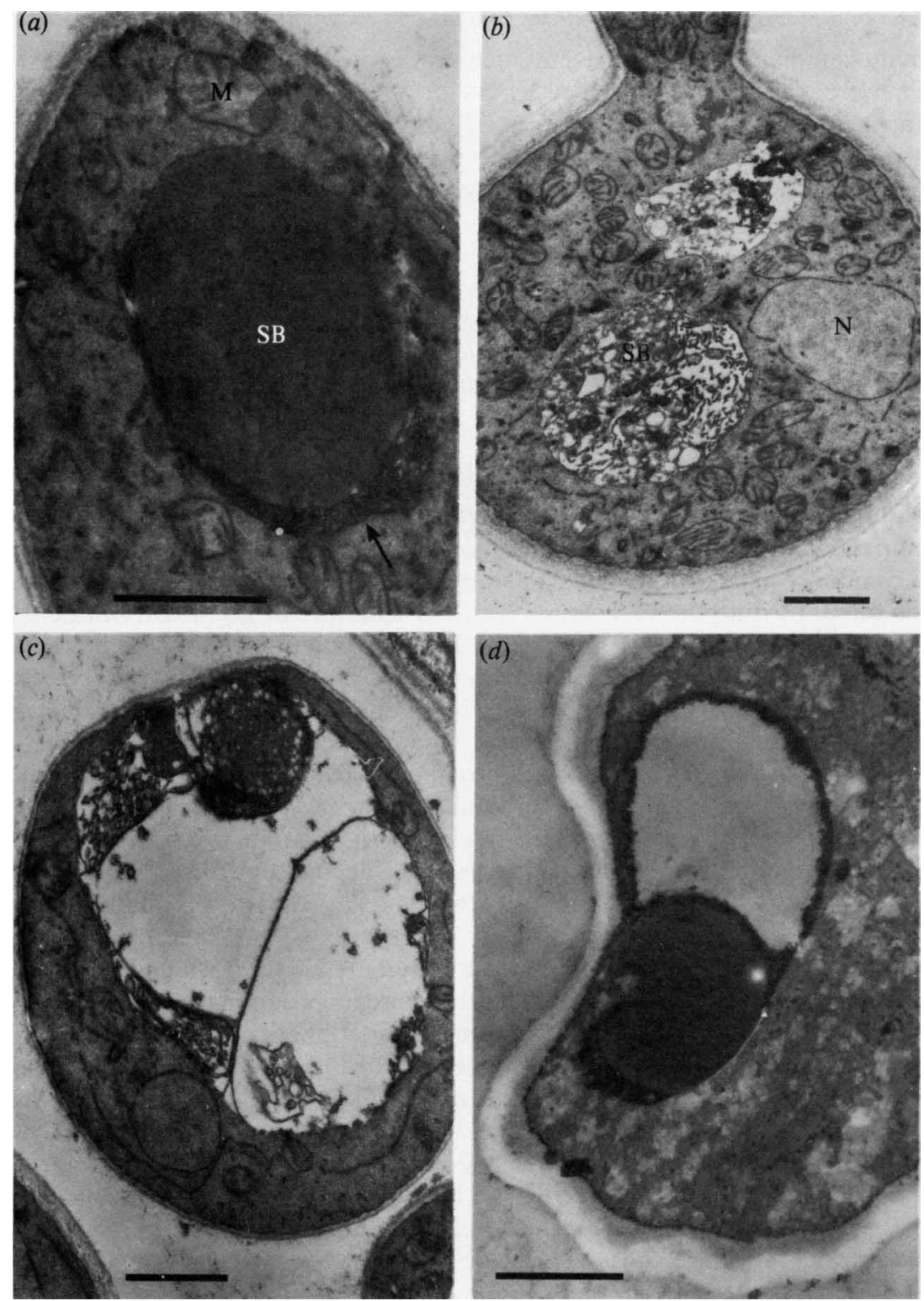

Fig. 3. (a) Storage body in a conidium. Note the compact membranous structure (arrow) associated with the dense inclusion substance. $(b)$ Longitudinal section of a germinating conidium. Two large storage bodies are interconnected with each other by their limiting membranes. (c) Cross section of a germ tube showing a large vacuole containing vesicles, debris, and remnants of stored substances. $(d)$ Demonstration of acid phosphatase in a dormant conidium. Heavy lead phosphate deposits are present in the vesicles. $\mathbf{N}$, nucleus; $\mathbf{M}$, mitochondrion; SB, storage body. The bar markers represent $1 \mu \mathrm{m}$.

38a (Fig. $1 b$ ). These isoenzymes, then, are present only in conidia. Among these isoenzymes specific to conidia, isoenzymes 3,4 and 5 were almost completely inactivated by heat treatment at $40^{\circ} \mathrm{C}$ for $20 \mathrm{~min}$ or by diisopropyl fluorophosphate treatment $\left(10^{-4} \mathrm{M}\right)$. All esterases were inactivated by treatment at $60^{\circ} \mathrm{C}$ for $20 \mathrm{~min}$, except for band 2 which retained some residual activity (Fig. 2). 


\section{Electron microscopy of germinating conidia and localization of acid phosphatase}

In dormant conidia of $N$. crassa, several inclusion bodies containing electron-dense granular compounds were observed, as described by Weiss (1963). From their staining properties and general appearance, these granules seem to be composed of lipids (Fig. $3 a$ ). In germinating conidia, the density of the inclusion bodies decreased, and they became coarse in appearance (Fig. $3 b$ ). This may indicate that the stored compounds are digested to supply energy and materials for conidial germination.

In elongated hyphae, large and numerous vacuoles were observed in which the electron-dense granules were scarcely visible (Fig. $3 c$ ). Complex lamellar and/or tubulous structures have been detected frequently in the vacuolization process of the storage bodies as described by Zalokar (1961) and Kozar \& Weijer (1969). Activity of acid phosphatase, which can hydrolyse $\beta$ glycerophosphate was detected within the storage bodies (Fig. $3 d$ ).

\section{DISCUSSION}

A large amount of the stored substances in dormant conidia may have an important role in early germination if associated with the digestion enzyme systems that can be readily activated as soon as conidia become hydrated under aerobic conditions. These endogenous substances are likely to be present within the vacuoles or vesicles (Schmit \& Brody, 1976; Matile, 1978). The accumulation in vesicles of basic amino acids and inorganic polyphosphate has already been described (Martinoia et al., 1979; Cramer et al., 1980). In addition, the electron-dense substances in vesicles have been accepted to be lipids or phospholipids (Weiss, 1963). Bianchi \& Turian (1967) estimated the content of lipids in conidia to be $17 \%$ of the dry weight. This high level of lipids is in keeping with the present observation that electron-dense bodies occupy the greater part of sectioned conidium (Fig. 3). Bianchi \& Turian (1967) also reported a remarkable decrease in lipid content between 3 and $5 \mathrm{~h}$ after inoculation, a time when active emergence and rapid elongation of germ tubes has been observed. The degradation of stored substances at this stage of conidial germination, as seen in Fig. $3(b)$ and $(c)$, is consistent with the data of Bianchi \& Turian (1967).

The evidence that conidia with high esterase activity tended to germinate rapidly indicates that stored ester compounds or lipids could be used for energy and material source in germ tube formation. However, there was not always a direct proportionality between the level of esterase activity and time of germination (compare culture 64 with 80 ). This fact indicates the possibility that other enzyme systems are also involved in the initial stage of germination, such as the trehalose-trehalase system (Hanks \& Sussman, 1969). The vacuoles or vesicles of Neurospora cells have been shown to contain many hydrolytic enzymes such as protease, RNAase, phosphoesterase, and invertase (Matile, 1971). In this study, the presence of acid phosphatase activity in vesicles has been shown (Fig. $3 d$ ). Because of the high activity of the above enzymes in the vesicles, the latter are considered to be lysosomes. Indeed, Wilson et al. (1970) have reported lysosomes quite comparable to animal lysosomes in several species of fungi. The inclusion bodies associated with membranous structures, shown in Fig. 3(a) and (b), closely resemble animal lysosomes. In spite of the lysosomic character of vesicles in conidia, it is possible, as suggested by Matile (1971), that their stored compounds and hydrolytic enzymes play an important role in the initiation of germination. The positive correlation between esterase activity and conidial germination supports this assumption.

Although an attempt to identify the isoenzyme(s) specific to conidial germination failed, the demonstration of esterase isoenzymes specific to dormant conidia (Fig. $1 b$ ) may provide a clue for the elucidation of the metabolism of ester compounds or lipids during germination. As seen in Fig. 2, those esterase isoenzymes (bands 3, 4 and 5) which were specific to conidia were different in physical and chemical properties from those that were also found in the hyphae (bands 2 and 6), i.e. they were easily inactivated by incubation at $40{ }^{\circ} \mathrm{C}$ for $20 \mathrm{~min}$ or by diisopropyl fluorophosphate treatment. Isoenzymes 3,4 and 5 were insensitive to $10^{-4} \mathrm{M}$-eserine sulphate (data not shown). Eserine sulphate inhibits choline esterase, while diisopropyl 
fluorophosphate inhibits both choline esterase and carboxyl esterase. Hence, isoenzymes 3, 4 and 5 belong to the carboxyl esterase group (B-esterase).

The author is indebted to Dr U. Takahama for discussions and helpful suggestions. Corrections to the manuscript were supplied by Dr C. S. Gowans.

\section{REFERENCES}

BARKA, T. \& ANDERSON, P. J. (1962). Histochemical methods for acid phosphatase using hexazonium pararosanilin as coupler. Journal of Histochemistry and Cytochemistry 10, 741-753.

Beadle, G. W. \& Tatum, E. L. (1945). Neurospora. II. Methods of producing and detecting mutations concerned with nutritional requirements. American Journal of Botany 32, 678-685.

Bianchi, D. E. \& Turian, G. (1967). Lipid content of conidia of Neurospora crassa. Nature, London 214, $1344-1345$.

Cramer, C. L., Vaughn, L. E. \& Davis, R. H. (1980). Basic amino acids and inorganic polyphosphates in Neurospora crassa: independent regulation of vacuolar pools. Journal of Bacteriology 142, 945-952.

Farach, M. C., Farach, H. \& Mirkes, P. E. (1979). Control of development in Neurospora crassa: nutrient requirements for conidial germ tube emergence, elongation, and metabolic activation. Experimental Mycology 3, 240-248.

Hanks, D. L. \& Sussman, A. S. (1969). The relationship between growth, conidiation and trehalase activity in Neurospora crassa. American Journal of Botany 56, 1152-1159.

Kozar, F. \& WeIJER, J. (1969). Electron-dense structures in Neurospora crassa. I. Mesosomes. Canadian Journal of Genetics and Cytology 11, 613616.

Markert, C. L. \& Faulhaber, I. (1965). Lactate dehydrogenase isozyme patterns of fish. Journal of Experimental Zoology 159, 319-332.
Martinoia, E., Heck, U., Boller, Th., Wiemken, A. \& Matile, P. (1979). Some properties of vacuoles isolated from Neurospora crassa slime variant. Archives of Microbiology 120, 31-34.

Matile, P. (1971). Vacuoles, lysosomes of Neurospora. Cytobiology 3, 324-330.

Matile, P. (1978). Biochemistry and function of vacuoles. Annual Review of Plant Physiology 29, 193213.

Mirkes, P. E. (1974). Polysomes, ribonucleic acid, and protein synthesis during germination of Neurospora crassa conidia. Journal of Bacteriology 117, 196-202.

Mirkes, P. E. (1977). Role of the carbon source in the activation of ribonucleic acid synthesis during the germination of Neurospora crassa conidia. Experimental Mycology 1, 271-279.

Schmit, J. \& BroDY, S. (1976). Biochemical genetics of Neurospora crassa conidial germination. Bacteriological Reviews 40, 1-41.

WeISS, B. (1963). An electron microscope and biochemical study of Neurospora crassa during development. Journal of General Microbiology 39, 85-94.

Wilson, C. L., Stiers, D. L. \& Smith, G. G. (1970). Fungal lysosomes or spherosomes. Phytopathology 60, 216-227.

ZaLOKAR, M. (1961). Electron microscopy of centrifuged hyphae of Neurospora. Journal of Biophysical and Biochemical Cytology 9, 609-617. 